\title{
Recent developments concerning the discovery of elements 101-111
}

\author{
Norman N Greenwood \\ School of Chemistry, The University of Leeds, Leeds LS2 9JT, England
}

\begin{abstract}
The addition of eleven new elements $(Z=101-111)$ to the Periodic Table during the past 40 years has involved outstanding feats of intellectual and experimental virtuosity. Some of the discoveries have been widely accepted but others have been hotly contested and this has led to distressingly persistent disagreements concerning priority. For this reason IUPAC and IUPAP (Physics) set up a joint Transfermium Working Group (TWG) in 1987 to recommend "the criteria that must be satisfied for the discovery of a new element to be recognised" and to apply these criteria to questions of priority in the discovery of the transfermium elements $(Z>100)$. After several years of detailed work the TWG published their extensive studies and conclusions, and these have been accepted by both IUPAC and IUPAP. Many subtle and difficult points were considered and these will be discussed in this lecture together with the TWG's final recommendations, which have been widely though not universally accepted by the scientific community. The separate question of names and symbols for the new elements with $Z>103$ has not yet been finally resolved but will be briefly discussed.
\end{abstract}

\section{INTRODUCTION}

The discovery of a new chemical element has always excited great interest throughout the ages. Considerable credit attaches to such discoveries and it is therefore not surprising that rival claims sometimes occur. In a sense, it does not matter to science in general who actually discovers each new element. But it does matter to those who perhaps spend the bulk of their scientific careers on such projects. Personal pride, institutional pride and even national pride become involved and hundreds of millions of dollars are invested. The main motivations are, of course, scientific: to gain a better understanding of nuclear structure and the forces which bind the subatomic particles; and to extend the chemical trends of the Periodic Table.

The problems I want to discuss in this article began some 35 years ago and developed eventually into a bitter cold-war dispute between scientists at Berkeley in the USA and Dubna in the USSR. An attempt was made to resolve this controversy by an international group set up in 1974 by IUPAC in collaboration with its companion organization IUPAP. The group consisted of three members from the USA, three from the USSR and three (including the chairman) from 'neutral' countries. For various reasons, partly related to its composition, this committee never completed its work, nor issued a report nor, indeed, ever met as a group, though there was some correspondence between members.

Twelve years later, in 1986, the Transfermium Working Group (TWG) was set up by IUPAP/C to determine first the criteria that must be satisfied for the discovery of a new chemical element to be recognised and then to apply these criteria to the discovery of the transfermium elements $(Z>100)$. It is perhaps worth noting in passing that the word discovery is something of a misnomer in this context: 'synthesis and characterization' of new elements would be a better description. The TWG consisted of nine senior scientist drawn from countries not directly involved in the disputes. They spent some thousands of hours over a period of five years, including seven week-long meetings in seven different countries, three of the meetings being held in the laboratories of chief concern. Their Reports were accepted by IUPAP and IUPAC and were published in two instalments in Pure and Applied Chemistry $(1,2)$ and as a whole elsewhere (3). 


\section{CRITERIA FOR DISCOVERY}

The criteria for discovery of a new chemical element proposed by the TWG (1) were widely disseminated and discussed before publication and were formally accepted by the three laboratories of main concern. It was recognised that very few properties (of which perhaps the only uncontentious example is the characteristic X-ray spectrum) are sufficient of themselves, in isolation, to establish the existence of a new element. For the rest, identification must rely on a combination of properties that will vary from case to case. Discovery of an element can be based on either chemical or physical evidence, or on both. The exact value of the atomic number $Z$ need not be determined, only that it is different from all $Z$ values observed before, beyond reasonable doubt. Chemical methods can yield excellent assignment criteria. Observations of analogies of chemical properties involving elements of unknown $Z$ with those of compounds of the same chemical type of known elements may suggest specific $Z$ assignments. Chemistry can be done with few or even single atoms of an element. In such cases, many repeated reactions take place with those few atoms. This occurs, for example, with methods like ion exchange, gas chromatography, gas thermochromatography and chemical vapour transport.

It follows from the diverse and multifold nature of the criteria for discovery that cases may arise where the discovery is incomplete or partial. Suppose that Paper I presents evidence relating to a possible new element which is not adequate of itself to establish the exixtence of the new element without the evidence published later in Paper II (perhaps from a different laboratory) that, together with Paper I, carries certainty but which is also not sufficient of itself. It is now certain that Paper I 'saw' the new element but could not prove it conclusively at the time. Where does the priority lie? Or should it be shared? And if it should happen that, between Papers I and II, Paper III was published, complete in itself and carrying conviction: does the credit lie wholly with III even though, after publication of II it is evident that I actually saw the new element first?

An even more subtle dilemma arises if Group A presents evidence not considered compelling at the time; Group B later presents compelling evidence; and still later Group A's evidence is generally recognised as being compelling after all. Where does the priority lie? Or again, Group X claims discovery of element $\mathrm{E}$ (falsely) with data later shown by another group to have been with certainty due to another new element $F$. A variant, which has also arisen, would be that Group Y reports data which they make the basis of no claim but which are later recognised as being the signals of a new element. Who made the element? Who made the discovery? And should any credit be accorded in discovery to someone who proposes and verifies a novel synthetic route which is later first successfully applied to a new element by someone else? Finally, in these questions of priority: what constitutes a publication? Must it be a dated, refereed paper published in a generally available journal? This would certainly exclude much excellent work which has had a profound influence on the subject and could even disadvantage the authors themselves if the referees or editors have insisted on the removal of some details for reasons of space or otherwise. Alternatively, could 'publication' include presentation of the work at an open international conference and subsequent publication as an edited report of the conference proceedings? And could a widely circulated prepublished laboratory report be accepted?

The TWG concluded that the circumstances of discovery of new elements are not always straightforward and that it is not always possible to assert confidently that a new element was discovered with the required certainty, by a certain group, using a certain method on a certain date. Often the situation is rather that relevant data which are accumulated over a period of time, perhaps years, perhaps in two or more laboratories, gradually bring the scientific community to the conviction that the existence of a new element has been established. The TWG made their own informed and dispassionate judgements on each of the elements concerned but recognised that others, particularly committed participants, might form other judgements. They were clear, however, that in some instances it would have been both unscientific and unjust to attempt to assign absolute priority to a single group in the 'discovery' of a new element. The sounder procedure, which they adopted in such cases, was to delineate and assess the evidence in each publication and to present an objective profile of discovery, indicating where possible the relative importance of the various steps. They also stated that confirmation was always desirable but not always realistically possible, and that otherwise well documented claims for priority should not be denied indefinitely simply for lack of independent confirmation.

\section{ROUTES TO THE TRANSFERMIUM ELEMENTS}

Two general types of nuclear reaction have been used to produce transfermium elements. The first type, hot fusion reactions, use accelerated light projectiles (typically ${ }_{5} \mathrm{~B},{ }_{6} \mathrm{C},{ }_{7} \mathrm{~N},{ }_{8} \mathrm{O}$ or ${ }_{10} \mathrm{Ne}$ ) to bombard actinide 
targets (typically ${ }_{92} \mathrm{U},{ }_{94} \mathrm{Pu},{ }_{95} \mathrm{Am},{ }_{96} \mathrm{Cm}$ or ${ }_{98} \mathrm{Cf}$ ). This method can be used effectively up to about $Z=$ 106 but increasingly the compound nucleus is formed with such high excitation energy that many particles, including charged ones, evaporate off before the desired product nucleus is reached. To solve this problem the group at Dubna suggested an alternative route, cold fusion, which exploits the fact that bombardment of nuclei such as ${ }_{82} \mathrm{~Pb}$ or ${ }_{83} \mathrm{Bi}$ (which have high binding energies due to closed nuclear shells) with moderately heavy ions which are preferably also near closed nuclear shells (e.g. ${ }_{24} \mathrm{Cr},{ }_{26} \mathrm{Fe}$ or ${ }_{28} \mathrm{Ni}$ ) at energies just above the Coulomb barrier produces compound product nuclei with much lower resultant excitation energies. As a result, the probability of (unwanted) fission is very much reduced and, under sufficiently fine-tuned conditions, neutron-only emission will dominate over all other light-particle emissions. This method has been outstandingly successful in producing elements with $Z>106$.

Little point would be served in attempting to condense the huge volume of fully referenced information and its assessment in the TWG's Reports $(2,3)$. Nor, indeed, would a bare repetition of the final conclusions do justice to the detailed arguments which led to these conclusions. Instead, a few of the points will be highlighted before going on to mention the most recent developments which have occurred during the past twelve months.

\section{Elements 101.102 and 103}

Mendelevium $(Z=101)$ was first made by the Berkeley group in 1955 by bombarding a tiny amount of ${ }_{9}$ Es with $\alpha$-particles, and was established with certainty in 1958 by further work at Berkeley. Nobelium $(Z=102)$ was first claimed in 1957 by an international group working in Stockholm but was not confirmed by work at Berkeley (1958); it was conclusively established by work at Dubna (1965) using reactions such as ${ }_{95} \mathrm{Am}\left({ }_{7} \mathrm{~N}, 4 \mathrm{n}\right){ }_{102}$ No. The discovery of element 103 , lawrencium, was even more protracted. An important step was made by the Berkeley group in 1961 but this fell short of full conviction. Papers from Dubna in 1965, 1968 and 1971 taken together approached effective certainty, but it was not until the Berkeley paper of 1971 that all reasonable doubt had been dispelled. In this case, with several papers of varying degrees of completeness and conviction and referring to several isotopes, the TWG concluded that full confidence built up over the course of a decade, with credit attaching to work in both Berkeley and Dubna.

\section{Elements 104.105 and 106}

Consideration of elements 104 and 105 was perhaps the most difficult part of the assessment work undertaken by the TWG and analysis of the papers claiming the discovery of these two elements occupied more than 10 pages of their Report $(2,3)$. In addition to questions of priority, the situation had been exacerbated by the extensive use in the literature of rival pairs of names for the two elements, none of which had been internationally agreed, or recommended by IUPAC. Although it is clearly unsatisfactory and potentially very confusing to have more than one name for any given element, questions of nomenclature and the naming of the elements fell quite outside the terms of reference and competence of the TWG and were not considered by them. (See last section of the present paper.)

The first (inconclusive) work bearing on the synthesis of element 104 was published by the Dubna group in 1964 and concerned the bombardment of a ${ }_{96} \mathrm{Pu}$ target (mixture of 3 isotopes) with ${ }_{10} \mathrm{Ne}$ : detection was by spontaneous fission. However, the crucial Dubna evidence (1969-70) for the production of element 104 came after the development of a sophisticated method for rapid chlorination of the product atoms and their gas-chromatographic separation on an atom-by-atom basis. This was a heroic enterprise which combined cyclotron nuclear physics and chemical separations. The actinide series of elements ends with ${ }_{103} \mathrm{Lr}$. The next element should be in Group 4 of the transition elements---a heavier congener of $\mathrm{Ti}, \mathrm{Zr}$ and Hf. As such it would be expected to have a (tetra)chloride which was significantly more volatile than those of the actinides. After extensive preliminary work at Dubna to develop and prove the method, the recoil products emitted from the target were chlorinated by interaction with a stream of gaseous $\mathrm{NbCl}_{5}$ or $\mathrm{ZrCl}_{4}$ within a fraction of a second from the instant of formation of the new atom, and then separated gaschromatographically in a 4 metre long quartz tube at either $250^{\circ}$ or $300^{\circ} \mathrm{C}$, before being detected by spontaneous fission. When part of the tube was replaced by a $\mathrm{KCl}$ capillary the activity in the detection zone ceased due to the formation of an involatile complex, presumably $\mathrm{K}_{2}[104] \mathrm{Cl}_{6}$. As no nucleus with $Z$ $>104$ can be formed by bombarding ${ }_{94} \mathrm{Pu}$ with ${ }_{10} \mathrm{Ne}$, and no spontaneously fissioning atom with $Z<104$ forms a volatile chloride, the new activity must be due to element 104 . The chromatographic method was not well suited to establishing an accurate half-life for the few events detected, but half-life itself is not an assignment criterion and it was shown that the results were consistent with the half-life later determined for ${ }^{259} 104$. During the latter stages of this work, and essentially contemporaneously with it, the Berkeley group established the reactions ${ }^{249} \mathrm{Cf}\left({ }^{12} \mathrm{C}, 4 n\right){ }^{257} 104,{ }^{249} \mathrm{Cf}\left({ }^{13} \mathrm{C}, 3 \mathrm{n}\right){ }^{259} 104$ and ${ }^{248} \mathrm{Cm}\left({ }^{16} \mathrm{O}, 6 \mathrm{n}\right){ }^{258} 104$ by elegant work which included observation of generic parent-daughter $\alpha$-decays to the known isotopes 
${ }^{253} 102$ and ${ }^{255} 102$. The TWG concluded that credit for the discovery of element 104 should be shared between the groups at Dubna and Berkeley.

Attempts at the synthesis of element 105 were first reported from Dubna in 1968 but it was a further two years before a combination of cyclotron physics, a thermal-gradient variant of gas chromatography, and parent-daughter $\alpha$-particle generic relations finally succeeded in convincingly establishing its formation (papers submitted for publication Feb-June 1970). The main reactions studied were ${ }_{95} \mathrm{Am}+{ }_{10} \mathrm{Ne}={ }^{261} 105$ $+4 \mathrm{n}\left(\right.$ and $\left.{ }^{250} 105+5 \mathrm{n}\right)$. During the later stages of this work the Berkeley group published a convincing synthesis via ${ }_{98} \mathrm{Cf}\left({ }_{7} \mathrm{~N}, 4 \mathrm{n}\right)^{260} 105$ (submitted in April 1970) which was also secured, amongst much other evidence, by an $\alpha$-particle generic relation with ${ }^{256} 103$. TWG concluded that the independent work from the two laboratories was essentially contemporaneous and that credit for the discovery of element 105 should be shared.

Element 106 is much more straitghtforward. Work at Berkeley-Livermore (1974) first convincingly demonstrated the synthesis of this element via ${ }^{249} \mathrm{Cf}\left({ }^{18} \mathrm{O}, 4 \mathrm{n}\right){ }^{263} 106$. The contemporaneous work at Dubna applied their novel cold fusion method to reactions such as ${ }_{82} \mathrm{~Pb}\left({ }_{24} \mathrm{Cr}, x \mathrm{n}\right)^{259} 106$ : though this method was crucial to the synthesis of all later elements (107-111) it did not at that time demonstrate the formation of element 106 with adequate conviction. Very recently, element 106 was resynthesized by a new group at Berkeley using the same reaction (4); the isotope ${ }^{263} 106$ decays with a half-life of 1 second to ${ }^{259} 104$ and then by a second $\alpha$-particle emission to ${ }^{255}$ No, both of which were also positively identified.

\section{Elements 107,108 and 109}

These three elements were all first synthesized by the cold fusion method at GSI, Darmstadt $(2,3)$ using a very sophisticated set of techniques. For element 107 (1981) an accelerated beam of ionized ${ }^{54} \mathrm{Cr}$ atoms was made to impinge on a thin ${ }^{209} \mathrm{Bi}$ foil; the reaction recoils were separated in flight from the incoming beam and from the unwanted products of transfer reactions by a velocity filter consisting of a combination of magnetic and electric fields. This facility is known by the acronym SHIP, i.e. separated heavy-ion reaction products. The product atoms were then implanted in position-sensitive solid-state detectors which recorded $\alpha$-particle decay energies or spontaneous fission events in position- and time-correlation with each other and with the time of implantation. Time-of-flight was also used to estimate the masses of these particles. Five atoms of ${ }^{262} 107$ were detected and characterized in this way.

Element 108 was unequivocally established in 1984 by detailed work at GSI using the SHIP facilities to detect three atoms of the element formed by the reaction ${ }^{208} \mathrm{~Pb}\left({ }^{58} \mathrm{Fe}, \mathrm{n}\right)^{265} 108$. Simultaneous and independent work at Dubna on similar reactions, though less detailed and compelling, also led to the formation of element 108.

Element 109 was discovered by the Darmstadt group in 1982 in an astonishingly virtuoso experiment which convincingly detected just one atom of ${ }^{266} 109$ from the reaction ${ }^{209} \mathrm{Bi}\left({ }^{58} \mathrm{Fe}, \mathrm{n}\right)$. A further two atoms were synthesized at GSI in 1988.

\section{Elements 110 and 111}

These two elements were first made towards the end of 1994 at GSI and so post-date the deliberations of the Transfermium Working Group. Nevertheless, the publications $(5,6)$ convincingly meet the stringent TWG criteria for discovery and have been widely accepted by the scientific community.

Initially, one atom of ${ }^{269} 110$ was detected and characterized on 9 November 1994 by the SHIP facilities at Darmstadt following the reaction ${ }^{208} \mathrm{~Pb}\left({ }^{62} \mathrm{Ni}, \mathrm{n}\right){ }^{269} 110$ and observation of the subsequent chain of four $\alpha$ decays (5):

$$
{ }^{269} 110 \rightarrow{ }^{265} 108 \rightarrow{ }^{261} 106 \rightarrow{ }^{257} 104 \rightarrow{ }^{253} \text { No. }
$$

A further three atoms of ${ }^{269} 110$ and their decay chains were observed during the next eight days, leading to an average half-life of $170 \mu \mathrm{s}$ (uncertainty: $+160,-60 \mu \mathrm{s}$ ). Subsequent work also identified a second isotope, ${ }^{, 71} 110$ (see Chem. \& Eng. News, March 13, 1995, pp. 35-40).

Element 111 was synthesized and characterized by the same group (6) using the analogous cold-fusion reaction, ${ }^{209} \mathrm{Bi}\left({ }^{64} \mathrm{Ni}, \mathrm{n}\right){ }^{272} 111$, followed by observation of up to five $\alpha$-decays which could be assigned to the chain:

$$
{ }^{272} 111 \rightarrow{ }^{268} 109 \rightarrow{ }^{264} 107 \longrightarrow{ }^{260} 105 \rightarrow{ }^{256} \mathrm{Lr} \rightarrow{ }^{252} \mathrm{Md} .
$$


In all, three atoms of ${ }^{272} 111$ were observed during the period 8-17 December, 1994 , leading to an average half-life of $1.5 \mathrm{~ms}$ (uncertainty: $+2.0,-0.5 \mathrm{~ms}$ ). Note also the production of new isotopes of elements 107 and 109 .

The synthesis of each of the elements $112-114$ is currently the object of intensive research by several groups. It is anticipated that these elements will be progressively more stable than those immediately preceding them, but severe technical problems still remain and no convincing claims for the synthesis of any of these or still heavier elements have yet been published. However, in addition to the "discovery isotopes" mentioned in the preceding pages many other isotopes of the transfermium elements have been synthesized, as summarized in Table 1, making a total of at least 68 transfermium isotopes known so far.

TABLE 1. Isotopes of the transfermium elements (1995)

\begin{tabular}{lccc}
$\boldsymbol{Z}$ (Discovered) & No. of isotopes & Mass range & $\boldsymbol{t}_{1 / 2}$ range \\
\hline $\mathbf{1 0 1}(1955)$ & 14 & $247-260$ & $3 \mathrm{~s}-27.8 \mathrm{~d}$ \\
$\mathbf{1 0 2}(1965)$ & 12 & $250-262$ & $250 \mu \mathrm{s}-58 \mathrm{~m}$ \\
$\mathbf{1 0 3}(1961-71)$ & 10 & $253-262$ & $650 \mathrm{~ms}-3.6 \mathrm{~h}$ \\
$\mathbf{1 0 4}(1969)$ & $\mathbf{9}^{\text {(a) }}$ & $253-262$ & $7 \mathrm{~ms}-1.1 \mathrm{~m}$ \\
$\mathbf{1 0 5}(1970)$ & 7 & $255-263$ & $1.3 \mathrm{~s}-34 \mathrm{~s}$ \\
$\mathbf{1 0 6}(1974)$ & 6 & $259-266$ & $3.6 \mathrm{~ms}-30 \mathrm{~s}$ \\
$\mathbf{1 0 7}(1981)$ & 3 & $261-264$ & $12 \mathrm{~ms}-440 \mathrm{~ms}$ \\
$\mathbf{1 0 8}(1984)$ & 2 & 264,265 & $80 \mu \mathrm{s}-1.8 \mathrm{~ms}$ \\
$\mathbf{1 0 9}(1982)$ & 2 & 266,268 & $3.4 \mathrm{~ms}-70 \mathrm{~ms}$ \\
$\mathbf{1 1 0}(1994)$ & 2 & $269,(271)$ & $170 \mu \mathrm{s}$ \\
$\mathbf{1 1 1}(1994)$ & 1 & 272 & $1.5 \mathrm{~ms}$
\end{tabular}

${ }^{(a)} \mathrm{A}$ tenth isotope, ${ }^{254} 104$, with hafe-life $0.5 \mathrm{~ms}$, is uncertain.

\section{NAMES AND SYMBOLS OF THE TRANSFERMIUM ELEMENTS}

The names and symbols of elements 101 (mendelevium, Md), 102 (nobelium, No) and 103 (lawrencium, Lr) are internationally agreed and secure. There is, unfortunately, no agreement on some of the other elements and rival names appear in the literature along national lines. The formal position is that the IUPAC Committee charged with the responsibility for recommending names and symbols for elements has published its proposals (7) but there has been some dissent, particularly from the USA and Germany and the matter is unlikely to be finally resolved before the next IUPAC General Assembly in 1997. In the meanwhile it seems prudent to eschew the use of trivial names but to refer to the elements punctiliously by their atomic numbers, as is done in this article. It should be recalled, however, that names approved by IUPAC are based on considerations of practicality and usage and carry no implications regarding priority of discovery (8). Indeed, it is not difficult to think of several elements in the Periodic Table whose names differ from those originally proposed by their discoverers. The names that are in current use (or so far proposed) for elements 104-111 are summarized in Table 2. The urgent need for consensus is apparent.

Table 2. Names and symbols in current use (or so far proposed) for elements 104-111 $1^{(a)}$

\begin{tabular}{llll}
\hline $\boldsymbol{Z}$ & Systematic (1977) & IUPAC (1994) & Other names suggested by national groups \\
\hline $\mathbf{1 0 4}$ & Un-nil-quadium (Unq) & Dubnium (Db) & Kurchatovium (Ku), Rutherfordium (Rf) \\
$\mathbf{1 0 5}$ & Un-nil-pentium (Unp) & Joliotium (Jl) & Nielsbohrium (Ns), Hahnium (Ha) \\
$\mathbf{1 0 6}$ & Un-nil-hexium (Unh) & Rutherfordium (Rf) & Seaborgium (Sg) \\
$\mathbf{1 0 7}$ & Un-nil-septium (Uns) & Bohrium (Bh) & Nielsbohrium (Ns) \\
$\mathbf{1 0 8}$ & Un-nil-octium (Uno) & Hahnium (Hn) & Hassium (Hs) \\
$\mathbf{1 0 9}$ & Un-nil-ennium (Une) & Meitnerium (Mt) & - \\
$\mathbf{1 1 0}$ & Un-un-nilium (Uun) & -- & - \\
$\mathbf{1 1 1}$ & Un-un-unium (Uuu) & -- & --
\end{tabular}

\footnotetext{
(a) The hyphens in the systematic names have been inserted here to assist comprehension and pronunciation;
} they are not part of the names. 


\section{REFERENCES}

1. A. H. Wapstra, D. H. Wilkinson, I. Ulehla, R. C. Barber, N. N. Greenwood, A. Z. Hrynkiewicz, Y. P. Jeannin, M. Lefort and M. Sakai. Pure \& Appl. Chem. 63, 879-886 (1991).

2. D. H. Wilkinson, A. H. Wapstra, I. Ulehla, R. C. Barber, N. N. Greenwood, A. Z. Hrynkiewicz, Y. P. Jeannin, M. Lefort and M. Sakai. Pure \& Appl. Chem. 65, 1757-1814 (1993). See also Responses on the Report by A. Ghiorso and G. T. Seaborg, pp. 1815-20; Yu. Ts. Oganessian and I. Zvara, pp. 1820-21; P. Armbruster, F. P. Hessberger, S. Hofmann, M. Leino, G. Münzenberg, W. Reisdorf and K.-H. Schmidt, pp. 1822-23; and the Reply to the Responses by members of the TWG, p. 1824.

3. R. C. Barber, N. N. Greenwood, A. Z. Hrynkiewicz, Y. P. Jeannin, M. Lefort, M. Sakai, I. Ulehla, A. H. Wapstra and D. H. Wilkinson. Progress in Particle and Nuclear Physics 29, 453-530 (1992)

4. K. E. Gregorich, M. R. Lane, M. F. Mohar, D. M. Lee, C. D. Cacher, E. R. Sylwester and D. C. Hoffman, Phys. Rev. Lett. 72, 1423-1426 (1994).

5. S. Hofmann, V. Ninov, F. P. Hessberger, P. Armbruster, H. Folger, G. Münzenberg, H. J. Schött, A. G. Popeko, A. V. Yeremin, A. N. Andreyev, S. Saro, R. Janik and M. Leino, Z. Phys. A 350, 277-280 (1995).

6. Idem, ibid., pp. 281-282.

7. IUPAC Commission on Nomenclature of Inorganic Chemistry, Pure \& Appl. Chem. 66, 2419-2421 (1994).

8. Nomenclature of Inorganic Chemistry: IUPAC Recommendations 1990, (The Red Book), G. J. Leigh (ed.), Blackwell Scientific Publications, Oxford, 1990, p. 35. 